\title{
TRADUIRE ANDRÉS SÁNCHEZ ROBAYNA PETIT JOURNAL D'UNE TRADUCTION
}

\author{
Jacques ANCET \\ jacques.ancet@gmail.com
}

20 octobre 2016

T'ouvre La sombra y la apariencia. J'aborde le premier poème de la première section intitulée «Initiale, ou fractures d'une impérieuse invitation ». J'en écris une première version rapide et libre :

Toi qui as aimé le soleil
et le centre, et toi qui désires
t'enfoncer dans la lumière,
la roche et la présence,
nues, invisibles,
et toi qui sur le sable
écoutes les pulsations
du corps et de la terre
visibles et invisibles,
dis aussi, entr'ouvertes
dans la lumière des mondes,
l'ombre et l'apparence.

Je constate qu'il est fait d'un dialogue et même d'une fusion entre rigueur formelle et cet insaisissable qui est l'objet du poème. Rigueur formelle comme tentative pour ordonner, ou mieux, donner forme au sans forme : les éléments et plus généralement le monde — à cette fuite de tout que suggèrent, précisément, comme deux lèvres entr'ouvertes, disparition, apparition, l'ombre et l'apparence. Cette rigueur formelle, ma version ne l'a pas, dans la mesure où c'est d'avantage le sens lexical qui m'a guidé. La métrique en est absente ou hasardeuse. Certains vers font écho à ceux de l'original, d'autres non. Il faut donc reprendre.

Car, ce qu'il faut c'est traduire ce double mouvement. Pour la rigueur formelle l'heptasyllabe assonancé - et dans d'autres poèmes de cette section, l'hendécasyllabe — , deux mètres hispaniques (celui des liras de Jean de la Croix) dont il faudra rendre la tension toute classique par un équivalent français, l'hexasyllabe (et le décasyllabe pour l'hendécasyllabe). Pour l'insaisissable, choisir autant que possible des mots "fuyants", comme ceux qui, dans leurs consonnes fricatives $(\mathrm{z} / \mathrm{c} / \mathrm{s}) \mathrm{créent,}$ 
autour de la résistance des occlusives (t/d, p/b), tels des rocs dressés, le chuchotement unanime du délitement de tout.

Le recours à l'hexasyllabe et au décasyllabe, plus courts et moins souples que les mètres originaux, oblige à certains choix. Le plus épineux est celui du premier vers : traduit littéralement il donne cet octosyllabe qu'il serait en espagnol sans les deux synalèphes qui le contractent tout en lui laissant son ampleur: «Toi qui as aimé le soleil »/ «Tú, que has amado el sol ». Après bien des tergiversations pour tenter de tout faire tenir dans un hexamètre (Toi, qui aimas soleil/et centre... Toi qui as aimé centre/et soleil ... ), j'en reste, pour le moment, à cet octosyllabe qui me parait être l'écho le plus fidèle au presque octosyllabe original. Seule dérogation pour tout le poème, mais dérogation minime, l'octosyllabe étant un mètre tout aussi classique que l'hexasyllabe, à la rigueur formelle qu'impose le poème. Donc :

Toi, qui as aimé le soleil
et le centre, et désires ...

traduction où le second «que » («qui ») est élidé mais sous-entendu...

\section{1 octobre 2016}

Mais, bien sûr, puisque la traduction est un travail sous contrainte, il faut s'en tenir à l'hexasyllabe comme l'original à l'heptasyllabe. Le problème du français, c'est qu'il n'a que des mots pluri-syllabiques (soleil / lumière) pour les deux monosyllabes clés que sont «sol » et «luz ». Il va donc falloir réduire. D'abord en jouant sur articles et prépositions. Ensuite, et c'est le moins satisfaisant, sur le temps du verbe : passé simple à la place du passé composé trop long:

Toi, qui aimas soleil et centre, qui désires

Sauf que le passé simple français a quelque chose de vieilli et que c'est le passé composé que l'usage courant privilégie. D'autant que l'espagnol qui l'utilise moins couramment, l'emploie ici. Donc : «Toi qui as aimé... ». Reste une syllabe. « Soleil » n'y tient pas mais « centre », oui. Alors que dans l'original, c'est l'inverse qui se produit: « centro » est trop long mais pas « sol». Le vers se fait avec toutes les contraintes de la langue et pas seulement celles du lexique. On peut alors risquer :

Toi, qui as aimé centre

et soleil, et désires

Je rétablis au passage le second « et » du deuxième vers car le balancement duel autour de l'axe $\mathrm{du}$ « $\mathrm{y} »$ est fondamental non seulement dans ce poème (sol y centro / roca y presencia / del cuerpo $\mathrm{y}$ de la tierra / la sombra y la apariencia) mais dans tout le livre dont le titre repose précisément sur cette opposition. 
Traduire Andrés Sánchez Robayna. Petit journal d'une traduction.

Pourtant l'absence du soleil en fin du premier vers me gêne. Je pense alors que la prononciation courante de «qui as aimé » réduit les deux syllabes de « qui a » à une seule "quia", ce qui permettrait de réintroduire « soleil » en fin de vers: « Toi qui as aimé soleil / et centre ».

La suite est plus facile, malgré «adentrarse» dont la traduction serait exactement «t'enfoncer ». Mais c'est trop long et « entrer» n'indique pas suffisamment la valeur de pénétration. D'où « plonger » et sa nuance d'enfoncement dans l'épaisseur matérielle :

plonger dans la lumière

la roche et la présence,

\section{2 octobre 2016}

Un petit problème à l'entrée de la deuxième strophe : «desnudas » qui compte trois syllabes pour son homologue français «nues » qui, cette fois, n'en compte qu'une. «Dénudées », par ses connotations trop corporelles, ne me satisfait pas. D'où le parti d'inverser l'ordre des mots du vers et, pour rendre l'idée de dépouillement, de traduire «desnudas» par «à nu », avec la présence du e muet à l'hémistiche : invincibl-e-za-nu. J'aurais pu, comme j'y ai d'abord pensé, rajouter un «et » entre les deux adjectifs («invincibles et nues »), conjonction qui, me semblait-il, se justifiait par le balancement duel autour $\mathrm{du}$ «y», dont je viens de parler. Mais je constate qu'à la présence symétrique des trois couples de substantifs à la fin de chaque strophe, correspond symétriquement deux couples d'adjectifs en début des deuxième et troisième strophes, lesquels ne sont pas réunis par une conjonction, mais séparés par une virgule. Le poème impose ordre, symétrie et limite, là où règne l'ouvert et l'illimité. Donc, une virgule dans les deux cas.

Second problème : au troisième vers, «écoutes » et son e muet devant «pulsations » (« latidos ») interdisant l'hexamètre, j'opte pour « entends », moins actif, mais qui conserve l'idée et qui a l'avantage de s'insérer dans le réseau des [ã] dont nous reparlerons :

entends les pulsations du corps et de la terre

L'inversion des termes au premier vers de la dernière strophe est réclamée, comme son symétrique de la seconde strophe («invincibles, à nu »), par la mesure de l'hexamètre, la synalèphe de «visibles, invisibles » lui ôtant une syllabe. D'où : «invisibles, visibles » où s'entend, comme précédemment le battement du $e$ muet : «invisi-bles-visibles ».

Vient ensuite le problème de la «luz», déjà mentionné, lequel ne peut être résolu «lumière » ayant trop de syllabes - que par l'emploi de «clarté » ou de «lueur ». A moins de supprimer «aussi »dans l'avant-dernier vers et de créer un enjambement dans la phrase (dis, entr'ouvertes, dans / la lumière des mondes), chose que ne se permet pas l'original dont le vers est toujours très régulier. Je choisis donc « lueur », pour l'instant. 
Dernière difficulté. Traduire «la sombra y la apariencia » par «l'ombre et l'apparence », c'est perdre une syllabe. Et «les ombres et l'apparence» ne fait pas l'affaire, la valeur générique et universelle d'ombre disparaissant dans le pluriel. Seule solution, légèrement redondante mais que justifie la multiplication déjà soulignée des «et » dans le poème : «et l'ombre et l'apparence ».

\section{5 octobre 2016}

J'ai essayé de suivre au plus près la somme de petits aménagements et/ou déplacements (et je ne les ai pas tous mentionnés) dont est fait un travail de traduction, s'il veut conserver la rigueur de l'original («le bon Dieu est dans le détail », disait Flaubert). Lequel se soumet lui-même, plus ou moins consciemment, à un certain nombre de contraintes imposées par la tradition et les lois du langage et de la langue dont je suis loin d'avoir rendu compte ici. Tels les réseaux signifiants comme, en premier lieu, celui de la syllabe « en », qui nous conduit de « centro » à « apariencia », ce couple d'opposés, en passant par la plongée dans la matière « adentrarse en », «presencia », « invencibles », «arena » à l'invocation finale « di también». Réseau auquel s'associe l'assonance de fin de vers (e/a) comme dans «presenci $a »$, aren $a »$, «entreabiertas» et «apariencia». Autant que la forme "audible" de ces échos, c'est leur forme moins "audible" mais tout aussi présente qu'il faut traduire. Ce «en » par exemple auquel fait écho, dans la traduction le [ãn] de «centre», «présence», «entends » (dont l'emploi se trouve pleinement motivé par rapport à «écoute »), «entr'ouvertes », « dans », «apparence ». Ou, autre exemple, mais il y en aurait d'autres, cette présence insistante de la consonne «s » initiale de «sol»/ «soleil», qui nous mène de ce même «sol » à l'ombre («sombra ») (même couple d'opposés que précédemment) par le maillage serré des pluriels, surtout, et de la deuxième personne du singulier, entre autres (deseas, presencia, desnudas, invencibles, sobre, escuchas, los, latidos, visibles, invisibles, entreabiertas); réseau auquel répond les [s] et les [z] de la traduction, non pas dans les pluriels (ils sont inaudibles) mais par les liaisons («qui a-zaimé », «invisible-za-nu», «di-zaussi ») ou un certain nombre de termes clés comme «centre», «présence », «sable », «pulsations », « invisibles », « visibles », créant un bourdonnement fuyant équivalent, me semble-t-il, à celui de l'original.

\section{6 janvier 2017}

Et, pour finir, le problème sans doute le plus difficile : celui de la traduction des assonances à chaque vers pair, qui méritent de trouver un équivalent dans la version française, ne serait-ce que pour faire entendre le léger écho traditionnel du poème original. Après bien des hésitations, c'est l'assonance [ãn] que je retiens, parce qu'elle participe du réseau dominant, comme l'assonance [e/a] associée au [en] dans l'original. Cette dernière me conduit à un certain nombre d'aménagements comme, par exemple, l'usage des gérondifs et l'enjambement dont il a été question plus haut que je me permets au deuxième vers de la dernière strophe pour conserver le mot « lumière » indispensable ici : 


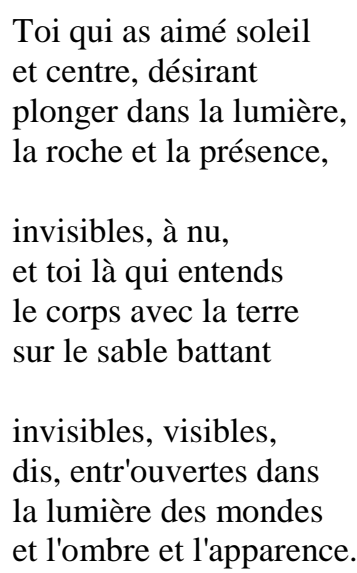

Sans doute cette version pourrait-elle être encore améliorée tant l'acte de traduire est une tâche infinie. Je ne vais donc pas plus loin, pareille analyse étant, elle aussi, infinie. Ce que j'ai voulu montrer par là, c'est l'extrême densité du poème qui est un objet verbal fait pour traverser le temps et auquel la traduction doit répondre par une densité analogue qu'elle doit conserver, même si elle aboutit nécessairement à un autre texte (une métaphore de l'original). C'est en cela qu'elle serait, comme on dit, "fidèle", et non en s'obstinant à traduire le seul sens des mots.

\section{Bibliographie}

SÁNCHEZ RoBAYNA, A. (2010): La sombra y la apariencia. Barcelona, Tusquets. 